\title{
Aminoaciduria en población infantil normal de 0 a 6 años
}

Dres. Cecilia Escobar*, Marta Colombo** y Kenneth Jones*; y senorita Luz Arévalo***.

La introducción de las técnicas de cromatografía en papel, ha permitido estudiar los aminoácidos, ya sea en plasma u orina, en forma relativamente sencilla. Sin embargo, en nuestro medio, esta técnica no se ha introducido en la práctica clínica, salvo para el caso de investigación de enfermedades metabólicas, en que es un examen de indiscutible valor.

En nuestro Servicio se montó este método de examen a fin de estudiar la eliminación urinaria de aminoácidos y analizar sus probables aplicaciones en la clínica pediátrica. Al revisar la bibliografia al respecto, no encontramos patrones nacionales normales para aminoaciduria en niños chilenos, y los datos de la literatura extranjera no nos fueron de gran ayuda, ya que, por un lado, existe total anarquía entre los diferentes autores en relación a la manera de expresar los resultados obtenidos ${ }^{4}$, por lo que no son en absoluto comparables entre sí $y$, por otro lado, no encontramos ningún autor que hubiera hecho mediciones en todos los grupos etarios.

*Servicio de Pediatría, Hospital Carlos van Buren, Valparaíso.

**Instituto de Nutrición y Tecnología de los Alimentos. Lniversidad de Chile, Sede Sur, Santiago.

*** Laboratorio Clínico Hospital Carlos van Buren, Valparaíso.

\section{OBJETIVOS}

1. Establecer nuestro propio patrón normal de aminoaciduria a diferentes edades pediátricas, que sirva de base a futuras investigaciones.

2. Comparar entre sí la eliminación de aminoácidos urinarios en los diferentes grupos etarios estudiados.

\section{MATERIAL}

Nuestro material consistió en 84 niños eutróficos y sanos entre 0 y 6 años, divididos en 3 grupos etarios (tabla 1).

Tabla 1
DISTRIBUGION POR EDADES DE 84 NINTOM EN QUIENES SE PRACTICO CROMATOGRAFIA UNIIDIMENSIONAL DE AMINOACIDOS URINARIOS
(Valparaiso, ('hile, 1976)

\begin{tabular}{lc} 
& 10 decasos \\
\cline { 2 - 2 } Recién nacidos & 14 \\
Lactantes & 27 \\
Preesolares & 43 \\
\hline
\end{tabular}


Esta muestra fue seleccionada del Consultorio Hospital de Niños de Valparaíso y de la Maternidad del sermena de Valparaíso.

Las condiciones para ser ingresados en este estudio fueron:

1. Buen estado nutritivo, y en el caso de los recién nacidos, ser de término, adecuado para la edad gestacional.

2. No presentar ningún tipo de patología.

3. No haber ingerido ningún tipo de medicamentos en las 48 horas previas al examen.

Las edades de los RN fluctuaron entre las 2 y 6 horas de vida, y en ninguno de los casos habían recibido algún tipo de aliment ación.

\section{METODO}

El análisis cromatográfico se efectuó en una muestra aislada de orina, tomada en cualquier hora del día, en un tubo limpio, lavado con agua destilada.

Se aplicó la técnica clásica de cromatografia unidimensional ascendente ${ }^{1}$, sembrando 10 lambdas de orina a estudiar y 10 lambdas de solución estándar, la cual se preparó con cantidades conocidas de los siguientes aminoácidos (tabla 2).

Tabla 2

ESTANDAR DE AMINOACIDOS

\begin{tabular}{ll}
\hline Leucina & Lisina \\
Fenil alanina & Histidina \\
Valina & Cistina \\
Tirosina & Glicina \\
Prolina & Serina \\
Alanina & Glutamina \\
Treonina & \\
\hline
\end{tabular}

Como soporte se usó papel Whatmann $3 \mathrm{~mm}$; como solvente, una mezcla de n-butanol-ac. acético; y como reactivo revelador, solución de ninhidrina al $0,2 \%$ en acetona.

Los aminoácidos de la muestra se identificaron por comparación con los del estándar, o por los valores $\mathrm{Rf}$. (El valor $\mathrm{Rf}$ es la relación entre la distancia recorrida por la sustancia y la distancia recorrida por el solvente: cada aminoácido tiene un Rf característico).

Para establecer la cantidad relativa de cada aminoácido, se tomó en cuenta el área de la mancha y la intensidad del color, comparándolo con diferentes diluciones del estándar de cada aminoácido por separado.

Para expresar los resultados en micromoles/ día, se tomó en cuenta el peso molecular del aminoácido y la diuresis diaria promedio para cada grupo etario.

\section{RESULTADOS Y COMENTARIOS}

En el grupo Recién Nacidos, se encontraron los siguientes resultados, que se expresan en valores promedios de micromoles/día (tabla 3).

\section{Tabla 3}

\section{EXCRECION URINARIA DE AMINOACIDOS EN 14 RN SIN RECIBIR ALIMENTACION, EXPRESADA EN MICROMOLES/DIA}

\begin{tabular}{lc} 
& Promedto \\
\cline { 2 - 2 } Glicina & 69 \\
Alanina & 21 \\
Serina & 57 \\
Treonina & 17 \\
Glutamina & 19 \\
Cistina & 14 \\
Lisina & 36 \\
Tirosina & 7,5 \\
Histidina & 27 \\
\hline
\end{tabular}

Hay que recalcar que la edad de estos RN variaba entre 2 y 6 horas de vida y en ningún caso habían recibido algún tipo de alimentación.

Los aminoácidos encontrados fueron 9, siendo el más altamente excretado la glicina, luego la serina, la lisina, etc., $y$, por último, la tirosina.

En el grupo de los lactantes se encontraron los siguientes resultados (tabla 4).

Tabla 4

EXCRECION URINARIA DE AMINOACIDOS EN 27 LACTANTES EXPRESAI)A EN MICROMOLES/DIA

\begin{tabular}{lc} 
& Promedio \\
\cline { 2 - 2 } Glicina & 259 \\
Alanina & 140 \\
Serina & 182 \\
Treonina & 110 \\
Glutamina & 110 \\
Cistina & 72 \\
Lisina & 110 \\
Tirosina & 49 \\
Histidina & 92 \\
\hline
\end{tabular}


Los aminoácidos excretados son los mismos que para el grupo RN, pero resulta evidente el aumento en las cantidades promedios excretadas, correspondiendo el valor más alto a la glicina, luego la serina, la alanina, etc., y, por último, la tirosina.

En el grupo preescolar, se encontraron los resultados resumidos en la tabla 5 .

Tabla 5

EXCRECION URINARIA EN 43 PREESCOLARES, EXPRESADO EN MICROMOLES/DIA

\begin{tabular}{lc} 
& Promertor \\
\cline { 2 - 2 } Glicina & 350 \\
Alanina & 256 \\
Serina & 240 \\
Treonina & 26.4 \\
Glutamina & 225 \\
Cistina & 155 \\
Lisina & 196 \\
Tirosina & 98 \\
Histidina & 169 \\
\hline
\end{tabular}

Los aminoácidos excretados son los mismos que los 2 grupos anteriores, siendo el aminoácido más altamente excretado la glicina, luego la treonina, la alanina, etc., $y$, por último la tirosina.

Los valores promedio han aumentado respecto a los grupos etarios anteriores.

Tabla 6

EXCRECION URINARIA IDE AMINOACIDOS EN 84 NIÑOS SEGLN EDAD, EXPRESADA EN MICROMOLES/DIA

\begin{tabular}{lccc}
\hline & $R \wedge$ & Lactante's \\
& $(16)$ & $\begin{array}{c}\text { Préescolares } \\
(24)\end{array}$ & $(4,3)$ \\
\cline { 2 - 4 } Glicina & 69 & 259 & 350 \\
Alanina & 21 & 140 & 256 \\
Serina & 57 & 182 & 240 \\
Treonina & 17 & 110 & 264 \\
Glutamina & 19 & 110 & 225 \\
Cistina & 14 & 72 & 155 \\
Lisina & 36 & 110 & 196 \\
Tirosina & 7,5 & 49 & 98 \\
Histidina & 27 & 92 & 169 \\
\multicolumn{4}{c}{ Se encontraron ocasionalmente cantidades mínimas de } \\
leucina, valina y fenil alanina.
\end{tabular}

\section{CONCLUSIONES}

1. Los aminoácidos excretados en la orina de los RN lactantes y preescolares son los mismos.

2. No habiendo recibido ningún recién nacido alimentación alguna, la aminoaciduria aumenta progresivamente con la edad, lo que no estaría de acuerdo con la literatura extranje$\mathrm{ra}^{6}$, en que se dar cifras mucho más elevadas precisamente en el grupo RN.

3. En los tres grupos etarios, el aminoácido más altamente excretado fue la glicina, lo que está de acuerdo con las cifras dadas en la literatura extranjera .

4. El aminoácido excretado en menor cantidad en los tres grupos etarios fue la tirosina, lo que no concordaría con cifras de la literatura extranjera ${ }^{4}$ en que varía entre cistina, fenil alanina, valina y tirosina, según el autor y el grupo etario.

5. El orden de los valores promedio intermedios entre glicina y tirosina, varía según el grupo etario.

\section{RESUMEN}

se estudió la climinación urinariá de aminoácidos en 84 niños normales, eutróficos y sanos, entre $0: 6$ años, separados en $14 \mathrm{RN}, 27$ lactantes y 43 preescolares.

Se utilizó la técnica de cromatografía en papel y los resultados se expresaron en micromoles/dia.

De ac uerdo con los resultados obtenidos, se concluye que.

a) La eliminación urinaria de aminoácidos aumenta progresivamente con la edad. (RN sin recibir alimentación.)

b) En los tres grupos etarios se eliminaron los mismos aminoácidos, siendo el más importante la glicina y el menos importante la tirosina, variando el orden de los demás, según el grupo etario.

\section{SLMMARY}

The urinary excretion of aminoacids studied was in 84 normal children bet ween 0 and 6 years of age: 14 ne wborns. 27 infants and 43 pre-school children.

The unidimensional Paper Chromatography Ternic was used and the results were expressed in micromols/day

According to these results we can conclude that:

a) Urinary excretion of aminoacids increases according to age.

b) The same aminoacids were excreted in the three differents age groups, glycine being the most important and tyrosine the least important in all groups. The order of the other aminoacids changes according to the group. 


\section{BIBLIOGRAFIA}

Smith, 1. Chromatographic Tecniques.

" Daudsen and Henry. Clinical Diagnosis by Laboratory Methods.

3. Snyderman, S.E. Ped. Clin of N.A., Feb. 1971; 199.
- Snyderman, S.E. and Holt, L.E. Advances in Pediatrics, Vol. 11, 1960; 209.

b Chisolm and Harrison. Ped. Clin. of N.A., Vol. 7, 1961); 333.

- Spitzer, A. Ped. Clin. of N.A., Vol. 18, May 1971; 377. 